Instructions for authors, subscriptions and further details:

\title{
http://mcs.hipatiapress.com
}

\section{Editorial: Paternidad y Cuidado en América Latina: Ausencias, Presencias y Transformaciones}

Francisco Aguayo ${ }^{1}$,

Gary Barker ${ }^{2}$

Eduardo Kimelman ${ }^{3}$

1) EME/Fundación CulturaSalud, Chile

2) Promundo-US, Estados Unidos

3) EME/Fundación CulturaSalud, Chile

Date of publication: June $21^{\text {st }}, 2016$

Edition period: June 2016 - October 2016

To cite this article: Aguayo, F., Barker, G. y Ekimelman, E. (2016). Paternidad y Cuidado en América Latina: Ausencias, Presencias y Transformaciones, Masculinities and Social Change,5(2),98-106. doi:10.17583/MCS.2016.2140

To link this article: http://doi.org/10.17583/MCS.2016.2140

\section{PLEASE SCROLL DOWN FOR ARTICLE}

The terms and conditions of use are related to the Open Journal System and to Creative Commons Attribution License (CC-BY). 


\section{Editorial: Paternidad y Cuidado en}

\section{América Latina: Ausencias, Presencias y}

\section{Transformaciones}

Francisco Aguayo

EME-Fundación CulturaSalud, Chile
Gary Barker

Promundo-US, Estados Unidos

Eduardo Kimelman

EME- Fundación CulturaSalud, Chile

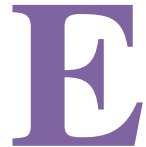

En América Latina hay cada vez más hombres que se interesan por participar en el cuidado y la crianza de sus hijas/os, especialmente los más jóvenes, con mayor escolaridad y aquellos con actitudes más equitativas de género (Barker \& Aguayo, 2011). Sin embargo, estos cambios se expresan fundamentalmente en el plano discursivo, y están muy lejos de la corresponsabilidad práctica y cotidiana entre hombres y mujeres en cuanto a la carga de cuidado y en la crianza. Hay hombres que se han ido incorporando en el ejercicio de una paternidad más activa, pero generalmente poniendo un mayor énfasis en ciertas actividades como el juego y en tareas de cuidado más ocasionales que habituales (Wainerman, 2007). Por otro lado, la paternidad se inscribe en relaciones de género y si bien más mujeres asumen que es importante la entrada de los hombres al campo doméstico y de la crianza, a algunas les ha costado ceder espacios que tradicionalmente se consideraron privativos de las mujeres (Valdés \& Godoy, 2008).

Las formas y roles dominantes de paternidad de los hombres se relacionan con la división sexual del trabajo lo que prolonga y refuerza la débil aproximación de los hombres al cuidado. En todos los países de América Latina la presencia de los hombres en el mercado del trabajo remunerado es mayor que la de las mujeres, así como -por el contrario- la presencia de las mujeres en el trabajo no remunerado -cuidado, crianza, tareas domésticas- es mayor que la de los hombres. Los estudios de uso de tiempo confirman que la suma de trabajo remunerado y no remunerado de 
las mujeres es mayor que el total de la suma de tiempo que destinan los hombres (CEPAL, 2014; CEPAL et al, 2014).

El rol proveedor sigue siendo un eje de la masculinidad hegemónica. Muchos hombres sienten que mantener a la familia y traer el pan a la casa es su principal cometido y tienen temor de no poder cumplir con ese mandato (Barker \& Aguayo, 2011; Olavarría, 2001). En América Latina solo un 3,2\% de los hombres en edad laboral reportan que están fuera del trabajo para atender tareas de cuidado y domésticas mientras que una de cada dos mujeres está en esa situación (CEPAL, 2014). De este modo en un gran porcentaje de familias observan todavía roles complementarios e inequitativos entre padre y madre. En estos casos el trabajo define la presencia de los padres con sus hijas/os la que ocurre fuera del horario de trabajo, los fines de semana, los días libres y en vacaciones. Por otra parte es creciente en la Región el porcentaje de familias en que padre y madre trabajan remuneradamente dada la mayor participación laboral de las mujeres (Ullmann, Maldonado \& Rico, 2014). En este tipo de arreglo hay mayores condiciones para la corresponsabilidad y la paternidad implicada (Rebolledo, 2008; Valdés \& Godoy, 2008), sin embargo, el tiempo que dedican los hombres al cuidado de los hijos en familias con dos proveedores económicos es menor que el de las mujeres (Aguayo, Correa \& Cristi, 2011). A pesar de que hoy cerca de 7 de cada 10 mujeres en edad reproductiva forman parte de la fuerza laboral los cuidados habituales continúan siendo una responsabilidad sobre todo de las mujeres (Blofield \& Martínez, 2014). El machismo y los roles tradicionales impiden avanzar hacia la corresponsabilidad en el cuidado de los hijos/as.

En la Región existe una gran cantidad de padres que están ausentes o no cumplen con sus responsabilidades económicas y o de cuidado. Esto tiene que ver con sus comportamientos reproductivos, con el trato diferenciado en la cultura a la maternidad y a la paternidad -los padres "pueden" desligarse no así las madres-, a la regla preferente de las madres para la tuición o custodia en caso de separación y con otros fenómenos como las migraciones. Por su parte los mecanismos para conseguir el pago de pensiones alimenticias son débiles en la mayoría de los países. Está pendiente investigar más sobre los costos económicos y sociales de la paternidad ausente y sobre cuáles son las barreras socioculturales y jurídicas que dificultan una mayor participación de los padres. 
Los cambios en las formas de paternidad de los hombres se relacionan con los cambios en los tipos de familias. La participación de los padres es mayor si residen con los hijos y disminuye una vez separados de la pareja, en medida importante porque aun los sistemas jurídicos y la tradición encargan la custodia a las madres y ubican a los padres como proveedores de visitas. Sin embargo, este sistema está en cuestionamiento por muchos padres e incluso madres. Un fenómeno creciente son las familias monoparentales a cargo de un papá que son cerca de 1 de cada 10 en la Región (Ullmann, Maldonado \& Rico, 2014). Frente a la desvinculación de la madre o su ausencia (en caso de muerte, migración, prisión, consumo de drogas o retiro voluntario de la maternidad) más padres deciden enfrentar su paternidad a solas y encuentran menos resistencias culturales o familiares para ello. Otro fenómeno creciente son las familias diversas homoparentales. Aquí se observan paternidades en familias con padres gay o trans. Se necesita avanzar en su reconocimiento social y legal, poder definir como padre a aquel de los dos que no es el genitor biológico, y facilitando el derecho a formar una familia a través de la adopción y la fertilización asistida cubierta por el estado.

La paternidad se relaciona con el machismo y las actitudes de género. En la Encuesta IMAGES en ciudades de Brasil, Chile y México se encontró que entre un $19 \%$ y un $35 \%$ de los hombres nunca se le enseñó a hacer tareas domésticas tales como preparar comida, limpiar la casa o el baño, lavar ropa o cuidar de hermanos pequeños. En cuanto a las tareas domésticas los hombres y las mujeres reportaron que los varones participan más en reparaciones en el hogar, pago de cuentas y compra de alimentos. Por otra parte su participación es débil en preparación de alimentos, limpieza del baño y lado de ropa. Las mujeres consideran que la participación de los hombres en esas tareas es menor que la que a su vez ellos reportan probablemente porque son ellas las que están a cargo de las tareas habituales. En Brasil y Chile solo una de cada 10 mujeres con hijos reportó que el padre se hacía cargo del cuidado diario de ellos. En los tres países la actividad con hijos de 0 a 4 años realizada con más frecuencia es el juego, más atrás preparar sus alimentos, cambiar pañales y bañarlos. Entre un $55 \%$ y un $61 \%$ de los hombres estuvo de acuerdo con la frase "tengo muy poco tiempo para estar con mis hijos por razones de trabajo", entre un $62 \%$ y un $80 \%$ estuvo de acuerdo con la frase "mi rol en el cuidado de los niños es principalmente como ayudante" (Barker \& Aguayo, 2011) 
Se necesita combatir el machismo en la paternidad y concientizar a los padres en la importancia de su rol y de compartir las tareas con la madre.

\section{¿Por qué es Importante Involucrar a los Papás en el Cuidado y la Crianza?}

Hay razones de diverso orden para promover una mayor participación de los padres en la crianza. La participación de los papás en el cuidado y crianza beneficia el desarrollo de sus hijas e hijos. Es creciente la evidencia que sugiere que cuando el papá está involucrado en la vida de sus hijas/os con una presencia de calidad esto tiene un impacto en su desarrollo, salud y bienestar en diversas áreas tales como mejores resultados en escalas de desarrollo cognitivo, mejor rendimiento escolar, menos problemas conductuales y menos conflictos con la ley (Levtov et al, 2015; Sarkadi et al., 2008). La participación de los hombres en el cuidado es clave para la equidad de género. Cuando los hombres comparten las tareas de cuidado residan o no con la madre- disminuye la sobrecarga de la madre, se mitigan las barreras para que la madre pueda realizar trabajo remunerado y se incrementa su bienestar. La paternidad implicada también impacta a los propios padres, quienes reportan sentirse más realizados y suelen cuidar más su salud (Barker \& Verani, 2008). La paternidad es también un asunto de derechos de las/os niñas/os a ser cuidados por ambos padres si los hay.

El tema de paternidad es una excelente puerta de entrada para el trabajo preventivo con hombres en violencia contra las mujeres y niñas y en violencia en género. Muchos hombres tienen interés en participar en actividades relacionadas con el desarrollo, salud y educación de sus hijas/os y el tema de paternidad les resulta atractivo y poco amenazante. Se necesita más oferta de programas de prevención de violencia que incluyan también la paternidad y el cuidado.

\section{¿Cómo se puede involucrar a los papás desde las políticas y programas?}

La paternidad puede ser promovida desde sectores como salud y educación que interactúan de forma regular con familias en sus prestaciones. Brasil y Chile destacan por sus acciones para promover la participación de los padres en/desde las prestaciones de salud. En Chile desde hace cerca de dos décadas se cuenta con una política de parto acompañado, llegando a un 


\section{Aguayo et al - Editorial}

$80 \%$ de los partos con acompañante en la actualidad, siendo en su mayoría el padre quien acompaña a la madre. También en un estudio reciente (Aguayo \& Kimelman, 2012) se encontró que en las ecografías en el sector público de salud la participación del padre es alta. En el contexto del Sistema de Protección a la Infancia chileno se ha desarrollado un programa de Paternidad Activa que busca orientar a los profesionales para promover la participación de los padres y disminuir las barreras para su incorporación en prestaciones tales como controles prenatales, parto, controles de salud 05 años, visita domiciliaria, talleres de habilidades parentales, entre otras (Aguayo \& Kimelman, 2012)

Para avanzar en el involucramiento de los padres en la crianza se necesita una adecuada oferta de talleres y programas socioeducativos. Experiencias como el Programa Paternidad Activa (CIDE, Chile), Programa H con hombres jóvenes (Brasil, México, Chile, entre otros) o el Programa $\mathrm{P}$ (Brasil, Chile, Nicaragua) con padres muestran que el dispositivo de taller en que los hombres comparten sus experiencias permiten instalar cambios de actitudes de género y promover comportamientos positivos. Se necesita una oferta de talleres sobre paternidad en contextos como los jardines infantiles, la escuela y salud, espacios habituales de vínculo entre los servicios y las familias. En el caso de salud los talleres de preparación al parto y de habilidades parentales son importantes tanto para preparar la llegada de un hijo como para apoyar la crianza.

Para promover la paternidad corresponsable se necesitan políticas orientadas a promover una mayor participación de los hombres la crianza y que ofrezcan mejores condiciones para resolver los dilemas que presentan el trabajo y el cuidado para cualquier hogar. Una política integral de paternidad y corresponsabilidad que busque transformar el actual orden de relaciones de género precisa de varios componentes: disminuir la brecha salarial, incrementar la participación de las mujeres en el trabajo remunerado, aumentar la cobertura de jardines infantiles, políticas de trabajo decente y conciliación laboral, permisos para que los papás puedan atender asuntos de salud o educación de sus hijas/os, postnatales masculinos largos intransferibles y pagados por el estado, fuero paternal, derecho a salas cuna en los lugares de trabajo, políticas de salud de los hombres, entre otras.

Los postnatales masculinos son una herramienta importante para involucrar a los hombres en el cuidado y la crianza. El debate sobre estos 
permisos es reciente en la Región. Las licencias para el padre son de duración escasa (Batthyány, 2015), usualmente como licencia pos-parto entre 1 y 14 días según el país. Más recientemente países como Chile han incorporado una licencia parental, en que la madre puede ceder parte de su permiso al padre. Sin embargo, en la experiencia chilena tan solo en un $0,3 \%$ de los casos el padre ha tomado la licencia (Lupica, 2015) lo cual se explica por razones culturales y también de su diseño ya que es un permiso transferible y coincidente con el período recomendado de lactancia materna. Los postnatales masculinos en la Región se encuentran en etapa embrionaria y aun no son vistos como una llave para avanzar en la incorporación de los hombres en el cuidado, la crianza y lo doméstico. Se necesita ampliar los permisos e instalar postnatales masculinos largos, con períodos intransferibles o cuota de paternidad que la experiencia comparada sugiere, constituyen el diseño que más promueve que los padres tomen efectivamente la licencia y tengan la experiencia de cuidar a tiempo completo. Por ejemplo, con 4 semanas que puedan tomarse después del nacimiento y otras semanas que puedan tomarse después de que la madre ha terminado su licencia. El debate sobre permisos iguales no ha llegado aun a la Región.

\section{Presentación de este Dossier sobre Paternidad y Cuidado}

El V Coloquio Internacional de Estudios de Varones y Masculinidades "Patriarcado en el siglo XXI: Cambios y Resistencias" se realizó en Santiago de Chile en enero de 2015. Fue organizado por la Fundación CulturaSalud/EME y la Universidad de Chile y fue patrocinado por la Alianza MenEngage, ONUMUJERES y UNICEF Chile.

Los temas de violencia y paternidad fueron aquellos con más ponencias recibidas. Se realizó un Grupo de Trabajo sobre Paternidad y Cuidado, algunas de las ponencias escritas se encuentran disponibles en www.coloquiomasculinidades.cl

Se incluyen en este dossier cuatro ponencias que fueron presentadas en el V Coloquio las cuales las cuales presentamos a continuación:

Juan-Guillermo Figueroa-Perea analiza las políticas públicas y programas de salud relacionados con comportamientos reproductivos de la población y demuestra cómo, a pesar de los discursos y orientaciones tendientes a la equidad de género, aquellas continúan centrándose en las 
mujeres e invisibilizando a los hombres, prolongando así el rol histórico reproductivo machista asignado a las mujeres y cerrando la incorporación de los hombres a esas áreas. Utilizando como insumo varias políticas públicas de salud reproductiva en México el autor ilustra con experiencias en esa línea, busca conceptualizaciones en torno a ellas y propone algunas líneas de trabajo para incorporar a los hombres.

Óscar Emilio Laguna-Maqueda presenta temas de paternidad ligados a la homosexualidad masculina. El autor hace visible por un lado las formas en que actualmente los hombres gay pueden acceder a ser padres y, como ello incide en la vivencia y el ejercicio de su paternidad. Por otro lado, analiza cómo los roles y arreglos parentales entre padres gay se vinculan con la noción tradicional de "familia", en qué medida la prolongan, la tensionan o la cuestionan.

Irene Salvo aborda el tema de la paternidad en el marco de la adopción. En el artículo se problematiza el lugar que se ha dado al hombre en la adopción, tanto en términos de genitor como de padre adoptivo. Se evidencian los patrones de roles dominantes que se mantienen y los temas específicos de paternidad que emergen de este tipo de experiencia.

Florencia Herrera y Yanko Pavicevic presentan parte de un proceso de investigación en curso que busca entender cómo cambió la paternidad en Chile en el tiempo reciente. Para ello se centran en pesquisar -mediante entrevistas- de qué manera los padres primerizos anticipan su paternidad, analizando los discursos de cómo los hombres se instalan y proyectan en el tránsito a su paternidad.

\section{Referencias}

Aguayo, F., Correa, P., and Cristi, P. (2011). Encuesta IMAGES Chile Resultados de la Encuesta Internacional de Masculinidades y Equidad de Género. Santiago: CulturaSalud/EME. Retrieved from https://www.researchgate.net/publication/258901222_Encuesta_IMAGE S_Chile_Resultados_de_la_Encuesta_Internacional_de_Masculinidades _y_Equidad_de_Genero

Aguayo, F., and Kimelman, E. (2012). Guía para promover la paternidad activa y la corresponsabilidad en el cuidado y la crianza de niños y niñas. Santiago: Chile Crece Contigo / Ministerio de Desarrollo Social. Retrieved from 
https://www.researchgate.net/publication/282672454_Guia_para_Promo ver_la_Paternidad_Activa_y_la_Corresponsabilidad_en_el_Cuidado_y_ la_Crianza_de_ninos_y_ninas

Barker, G and Verani, F. (2008). La participación del hombre como padre en la región de Latinoamérica y el Caribe: Una revisión de literatura crítica con consideraciones para políticas. Promundo / Save de Children. Brasil. Retrieved from http://promundoglobal.org/wpcontent/uploads/2015/01/La-participacion-del-hombre-como-padre-enla-region-de-Latinoamerica-y-el-Caribe.pdf

Barker, G. and Aguayo, F. (coords.) (2011). Masculinidades y políticas de equidad de género: Reflexiones a partir de la Encuesta IMAGES y una revisión de políticas en Brasil, Chile y México. Rio de Janeiro:

Promundo.

Batthyány, K. (2015) Las políticas y el cuidado en América Latina. Una mirada a las experiencias regionales. Asuntos de Género 124. Retrieved from

http://repositorio.cepal.org/bitstream/handle/11362/37726/S1500041_es. pdf? sequence $=1$

Blofield, M. and Martínez, J. (2014). Trabajo, familia y cambios en la política pública en América Latina: Equidad, maternalismo y corresponsabilidad. Revista de la Cepal 114, 108-125. Retrieved from http://repositorio.cepal.org/bitstream/handle/11362/37439/RVE114Blofi eldMartinez_es.pdf

CEPAL. (2014). La medición del tiempo y el aporte de las mujeres a la economía. Notas para la igualdad $\mathrm{N}^{\circ} 15$. Retrieved from http://www.cepal.org/oig/noticias/noticias/3/54223/Nota_para_la_iguald ad_N\%C2\%B015_-_EUT.pdf

CEPAL, FAO, ONU Mujeres, PNUD, OIT. (2013). Trabajo decente e igualdad de género. Políticas para mejorar el acceso y la calidad del empleo de las mujeres en América Latina y el Caribe. Retrieved from http://www.ilo.org/wcmsp5/groups/public/---americas/---ro-lima/---srosantiago/documents/publication/wcms_229425.pdf

Levtov, R., Van der Gaag, N., Greene, M., Kaufman, M., and Barker, G. (2015). State of the World's Fathers: A MenCare Advocacy Publication. Washington, DC: Promundo, Rutgers, Save the Children, Sonke Gender Justice, and the MenEngage Alliance 
Lupica, C. (2015). Corresponsabilidad de los cuidados y autonomía económica de las mujeres: Lecciones aprendidas del Permiso Postnatal Parental en Chile. Santiago de Chile: CEPAL, Serie Asuntos de Género. Naciones Unidas. Retrieved from http://repositorio.cepal.org/bitstream/handle/11362/37878/S1500262_es. pdf

Olavarría, J. (2001). Y todos querían ser (buenos) padres. Santiago de Chile: FLACSO. Retrieved from http://www.memoriachilena.cl/archivos2/pdfs/MC0056018.pdf

Rebolledo, L. (2008). Del padre ausente al padre próximo. Emergencia de nuevas formas de paternidad en el Chile actual. En Araujo, K. \& Prieto, M. (Eds.). Estudios sobre sexualidades en América Latina (pp.123-140). Quito, Ecuador: FLACSO.

Sarkadi, A., Kristiansson, R., Oberklaid, F. and Bremberg, S. (2008). Fathers' involvement and children's developmental outcomes: a systematic review of longitudinal studies. Acta Pcediatrica 97, 153-158. Retrieved from http://www.ncbi.nlm.nih.gov/pubmed/18052995

Ullmann, H., Maldonado, C. and Rico, M.N. (2014) La evolución de las estructuras familiares en América Latina, 1990-2010. Los retos de la pobreza, la vulnerabilidad y el cuidado. Santiago de Chile: CEPAL. Retrieved from http://repositorio.cepal.org/bitstream/handle/11362/36717/S2014182_es. pdf? sequence $=1$

Valdés, X. and Godoy, C.G. (2008). El lugar del padre: rupturas y herencias. Representaciones de la paternidad en grupos altos, medios y populares chilenos. Estudios Avanzados 6(9), 79-112. Retrieved from http://menengage.org/wp-content/uploads/2014/06/lugar-del-padre.pdf

Wainerman, C. (2007). Conyugalidad y Paternidad ¿Una revolución estancada? En Gutiérrez, M.A (Ed.). Género, familias y trabajo: Rupturas y continuidades. Desafíos para la investigación política (pp. 179-222). Buenos Aires: CLACSO. Retrieved from http://bibliotecavirtual.clacso.org.ar/ar/libros/grupos/gutierrez/09Wainer man.pdf 


$$
\text { MCS - Masculinities and Social Change, 5(2) } 107
$$

Francisco Aguayo es Investigador y Director de EME/Fundación CulturaSalud, Chile.

Gary Barker es presidente de Promundo-US, Estados Unidos.

Eduardo Kimelman es sociológo e investigador de EME/Fundación CulturaSalud, Chile

Contact Address: Direct correspondence to Francisco Aguayo, Almirante Pastene 12 of $\cdot 3 B$, Providencia, Santiago, Chile CP 7500551 email: faguayo@eme.cl 